\title{
Correlation of Parental Perception and Its Components with Attachment Style and Tendency to Communicate with the Opposite Sex in Veterans' Daughters
}

\section{ART ICLE INF O}

\section{Article Type}

Descriptive Study

\section{Authors}

Bazoolnejad M. ${ }^{1} M A$

Robatmili S.* PhD

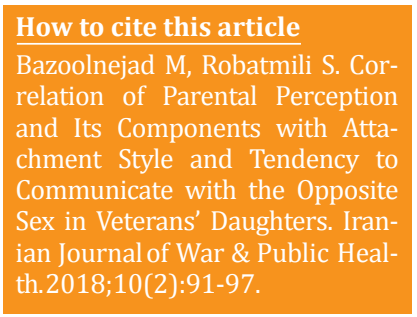

*Psychology Department, Humanities \& Social Sciences Facualty, Science and Research Branch, Islamic Azad University, Tehran, Iran ${ }^{1}$ Psychology Department, Humanities \& Social Sciences Facualty, Science and Research Branch, Islamic Azad University, Tehran, Iran

\section{Correspondence}

Address: Faculty of Humanities,

Science and Research Branch,

Islamic Azad University, Shahdia

Hesarak Blvd, Daneshgah Square,

End of Shahid Sattari Highway,

Tehran, Iran. Zip Code 1464785581

Phone: +98 (21) 44865216

Fax: -

somayemili@yahoo.com

\section{Article History}

Received: July 18, 2017

Accepted: November 21, 2017

ePublished: April 10, 2018

\section{A B S T R A C T}

Aims One of the factors damaging the family structure is the disability of a family member. Disability can be caused by events such as war; it may sometimes affect the senior family member, the father, and the children are also affected by this problem. The present study was conducted to evalualte the correlation of parental perception and its components with attachment style and tendency to communicate with the opposite sex in veterans' daughters. Instruments \& Methods In this descriptive-correlational study, 77 veterans' daughters in Tehran, Iran were selected by voluntary sampling method. The research tools consisted of Parental Bonding Instrument (PBI), Adult Attachment Questionnaire (AAQ), and Tendency to Opposite Sex Questionnaire. The data were analyzed by SPSS 23, using Pearson correlation coefficient test.

Findings There was a positive correlation between parental perception $(r=0.80)$ and the components of attempts to dependency ( $\mathrm{r}=0.87)$ and emotional relationship ( $\mathrm{r}=0.91)$ with tendency to the opposite sex, and there was a negative correlation between the components of indifference $(\mathrm{r}=-0.90)$ and encouragement for independence ( $\mathrm{r}=-0.84)$ with tendency to the opposite sex. Also, the attachment style and parental perception ( $\mathrm{r}=-0.85)$ had a negative correlation with the components of attempts to dependency $(r=-0.92)$ and emotional relationship ( $\mathrm{r}=-0.93)$, and had a positive correlation with the components of indifference $(r=0.92)$ and encouragement for independence $(r=0.89)$. Attachment style and tendency to the opposite sex $(\mathrm{r}=-0.92)$ had a negative correlation $(\mathrm{p}<0.01)$, too.

Conclusion Parental perception has a negative correlation with attachment styles and a positive correlation with the tendency to communicate with the opposite sex.

Keywords Parental Perception; Opposite Sex; Attachment Styles; Children; Veterans

\section{I T A T I O N L I N K S}

[1] The relationshib between marital values and reliegion oreiantation ... [2] Adolescent marital expectations and romantic ... [3] Comparison of relationship between religious attitude and paternal ... [4] Quality of value systems and self-efficacy beliefs ... [5] Childhood behavior problems linked to sexual risk taking in young ... [6] Correlates of premarital relationships among unmarried youth in ... [7] Investigating the role of family in communication ... [8] Values, Attitudes and Models of the Youth Actions in Choosing ... [9] The relationship between parental attachment ... [10] Parental attachment and love language ... [11] Perceptions about parents' relationship and parenting ... [12] Good Partner, good Parent responsiveness mediates ... [13] Parent-child relationship trajectories during adolescence: Longitudinal ... [14] Paternal influences on daughters' heterosexual relationship ... [15] Relationship of attachment styles and emotional intelligence ... [16] Attachment Styles in Sufferers of Gender Identity ... [17] Relationship between parenting styles and attachment ... [18] Rooting of prevalence of early relationship between ... [19] The effectiveness of conflict resolution skills in tends ... [20] Metaanalysis results of research on the mental health ... [21] Studying the role of family in communication ... [22] Statistics and research ... [23] Comparison of parenting pattern ... [24] Sensory processing sensitivity and its relation ... [25] The relationship between perceptions of parental ... [26] Attachment styles, illness perception ... [27] The relationship between attachment ... [28] Factors related to student's tendency ... [29] Change of values in cinema and ... [30] Wanting for others and sociology ... [31] The attitudes children of sacrificer and ... [32] Survey the effect of Lazarus's ... [33] Attachment quality and psychopathological ... [34] Maternal and paternal parenting styles ... [35] Attachment and loss: Separation ... [36] Rewriting family scripts: Improvisation and ... [37] Implications of inadequate parental bonding ... [38] Within-family conflict behaviors as ... 
در اثر حوادثى همجون جنگ ايجاد شده باشد كه گَاه عضو فعال،

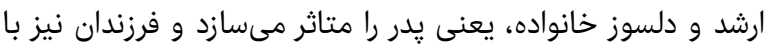

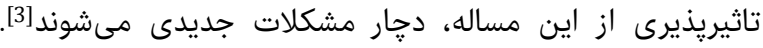

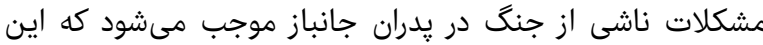

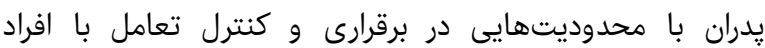

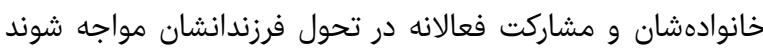

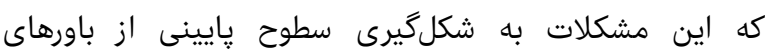

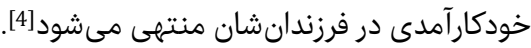

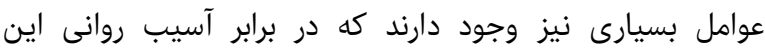

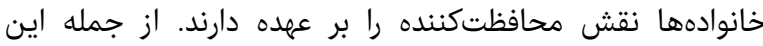

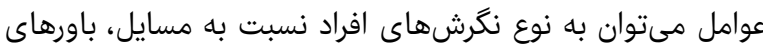

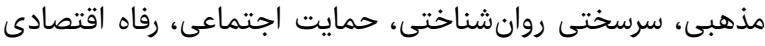

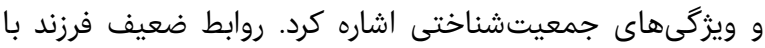

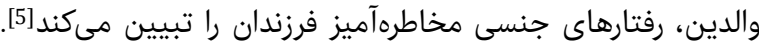

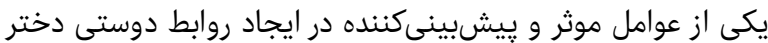

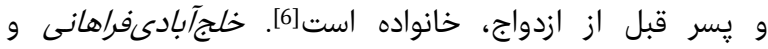

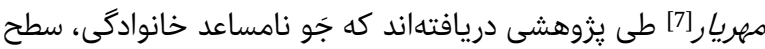

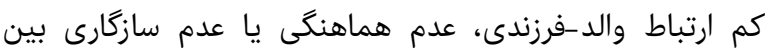

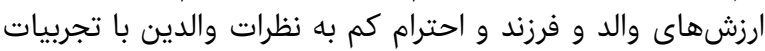

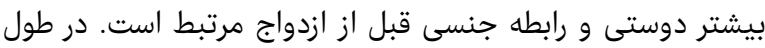

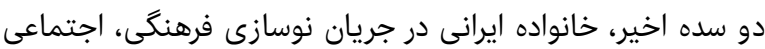

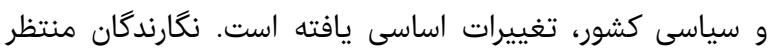

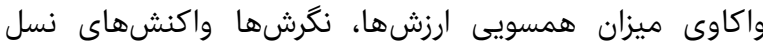

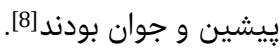

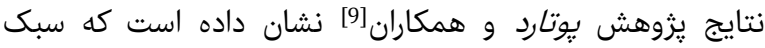

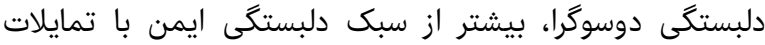

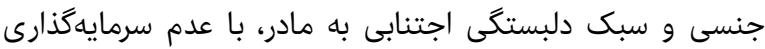

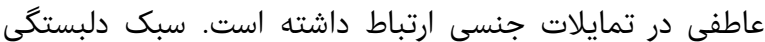

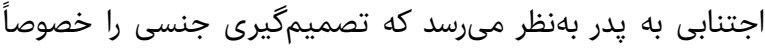

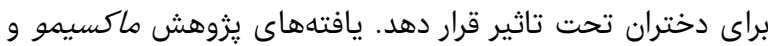

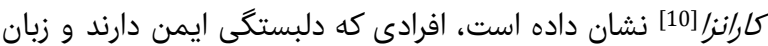

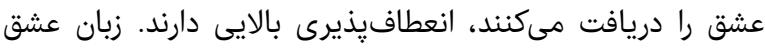

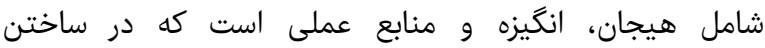

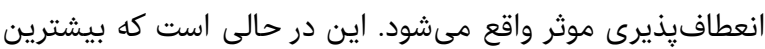

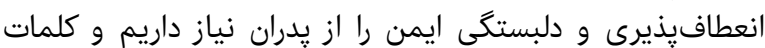

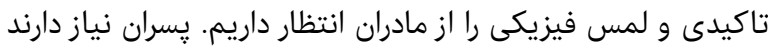

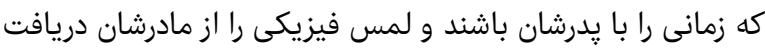

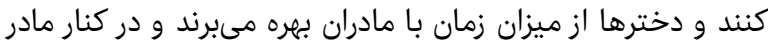

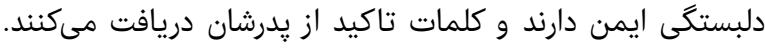

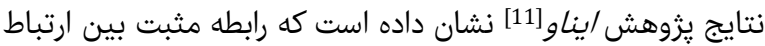

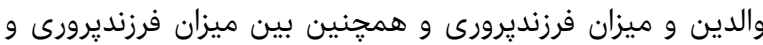

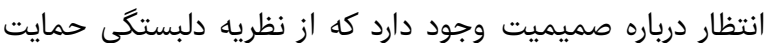

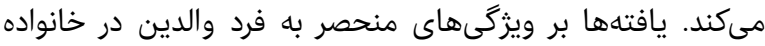

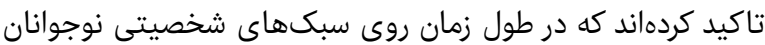

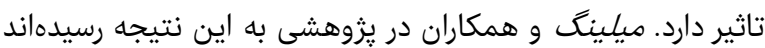

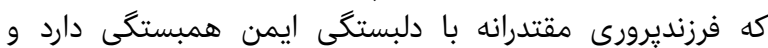

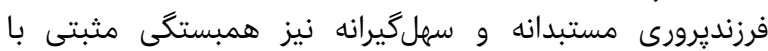

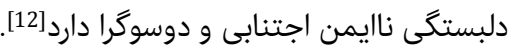

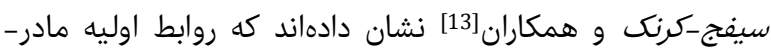

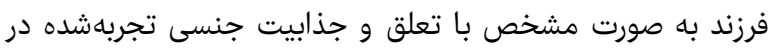

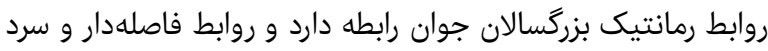
يدر-فرزند در طول نوجوانى با عشق اضطرابى رابط بعدى فرزندان روابط راصل وابطه همبستگى ادراك از والدين و مولفههاى آن بان

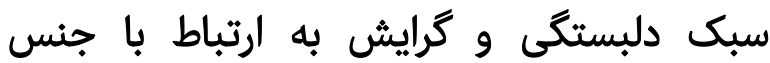
مخالف در دختران افراد جانباز

مرجان بذولنزراد MA

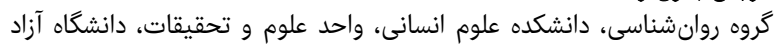
اسلامى، تهران، ايران

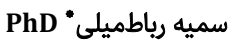

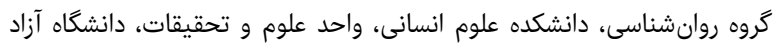

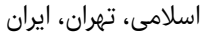

جكيده

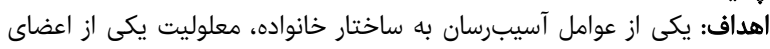

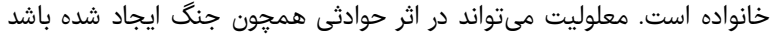

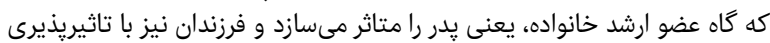

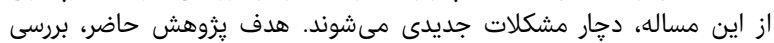

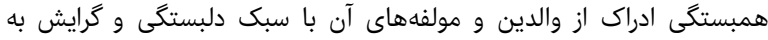

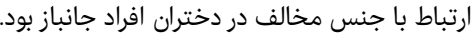

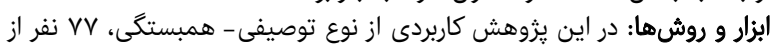

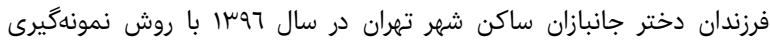

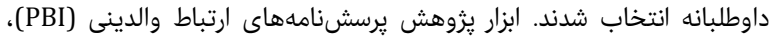

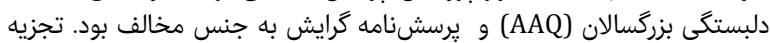

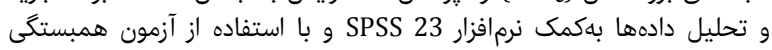

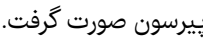

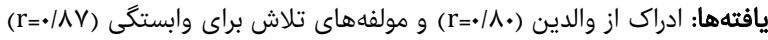

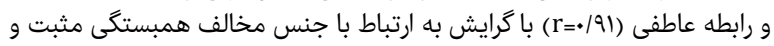

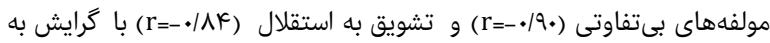

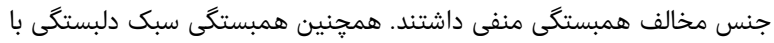

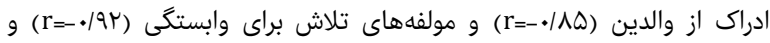

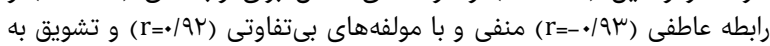

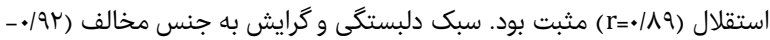

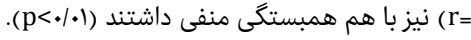

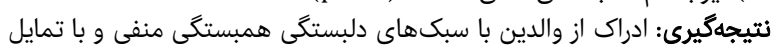

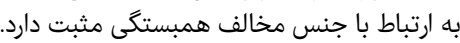

كليدوازهها: ادراك از والدين، جنس مخالف، همبسى مبكهاى دارد دلبستكى، فرزندان، جانبازان

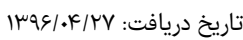

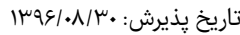

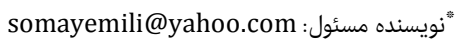

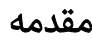

خانواده در طول تاريخ حيات بشرى به عنوان يك نهاد اجتماعى، درو دروز

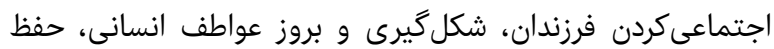

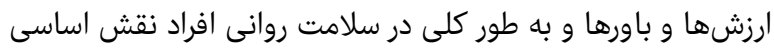

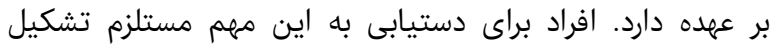

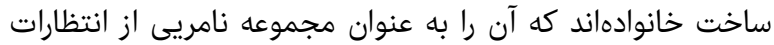

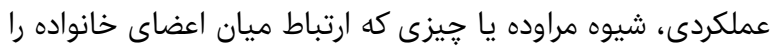

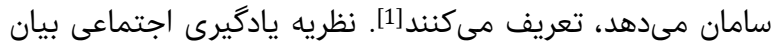

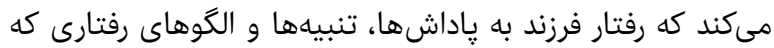

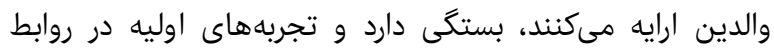

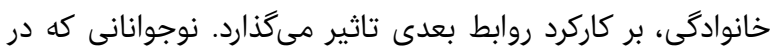

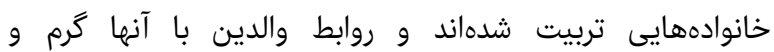

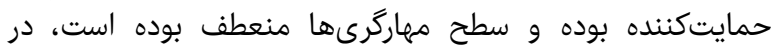

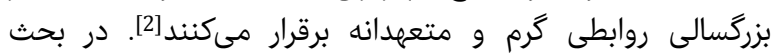

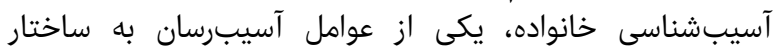
خانواده، معلوليت يكى از اعضاى خانواده است. معلوليت مىتواند آندان 


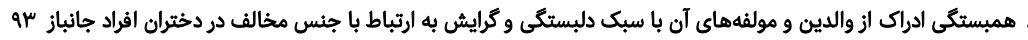

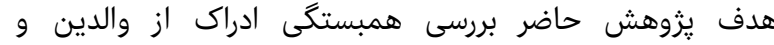

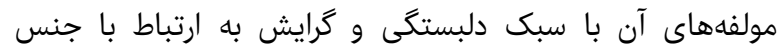
مخالف در دختران افراد جانباز بود.

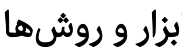

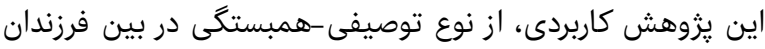

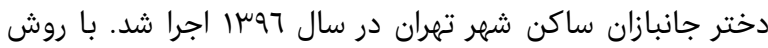

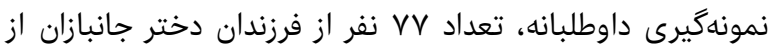

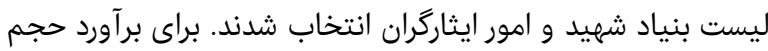

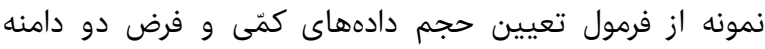

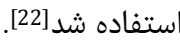

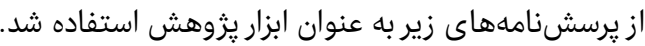

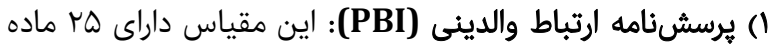

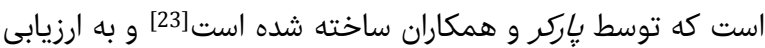

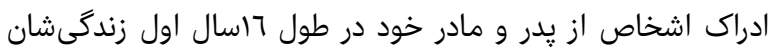

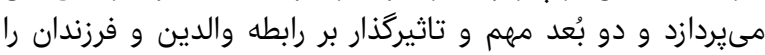

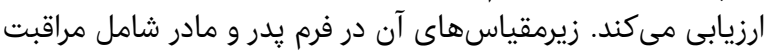

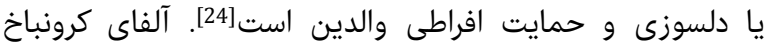

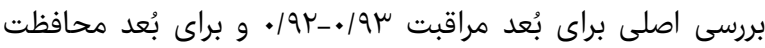

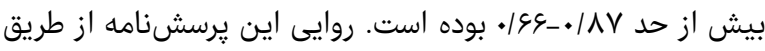

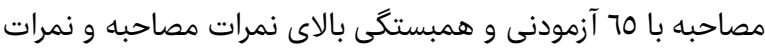

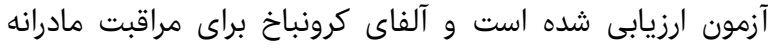

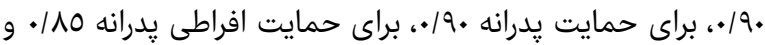

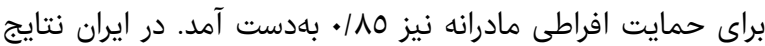

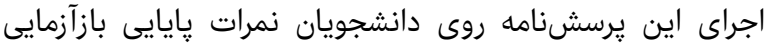

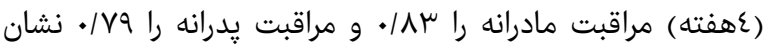

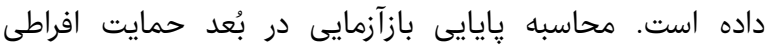

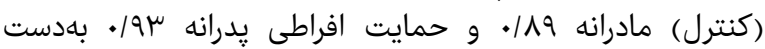

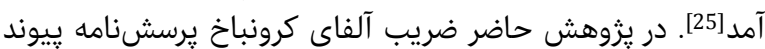

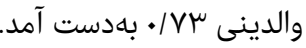

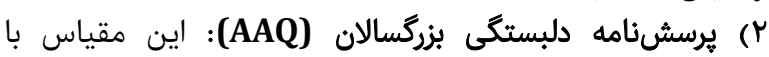

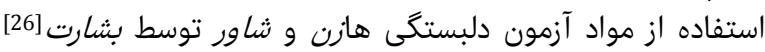

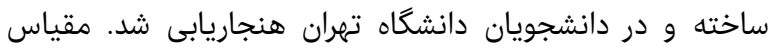

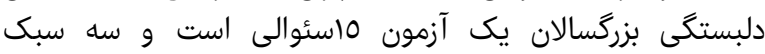

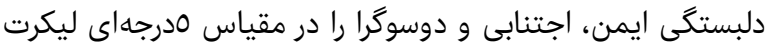

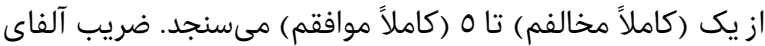

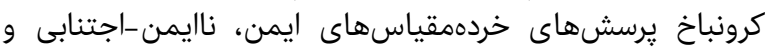

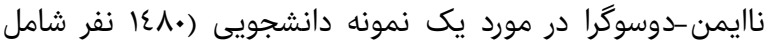

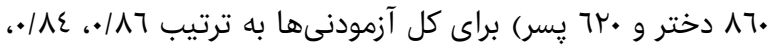

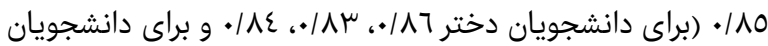

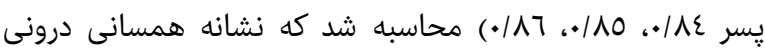

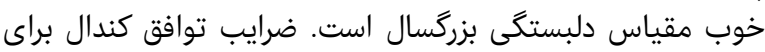

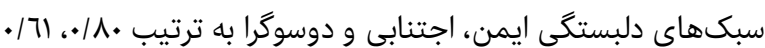

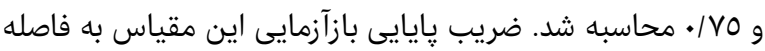

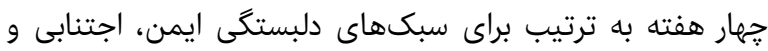

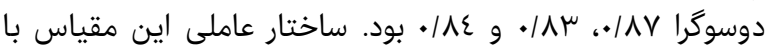

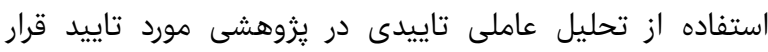

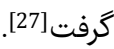

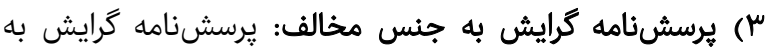

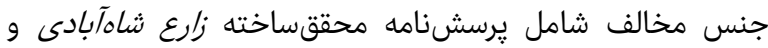

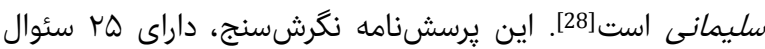

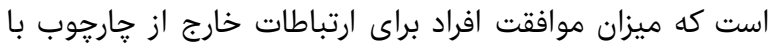

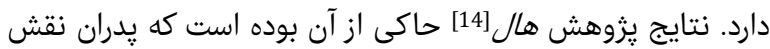

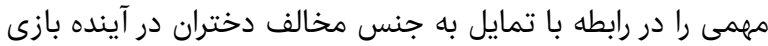

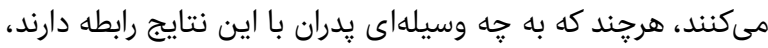

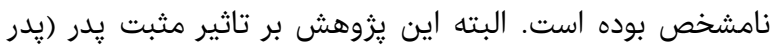

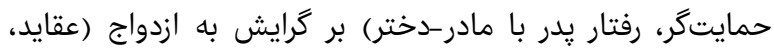

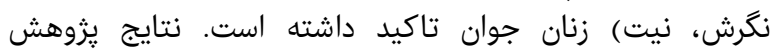

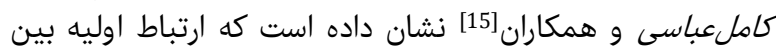

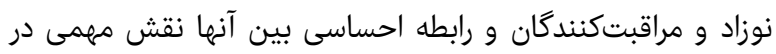

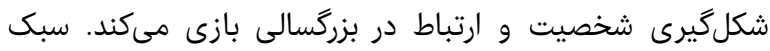
دلبستخى ايمن با رضايت زناشويى رابطه مثبت و و معنى دارئ دار داشته

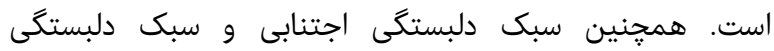

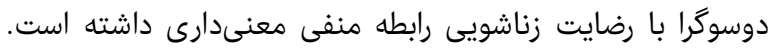

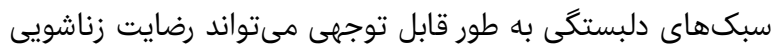

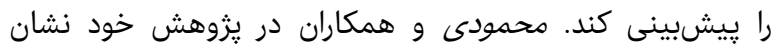

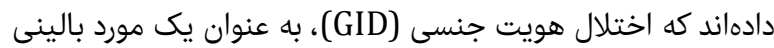

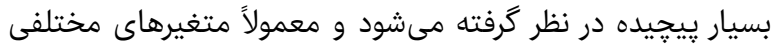

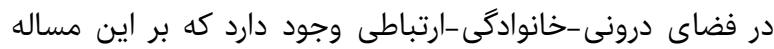

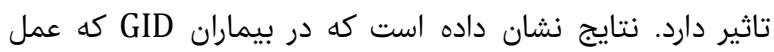

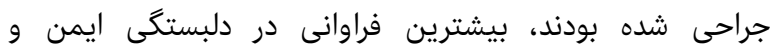

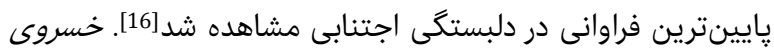

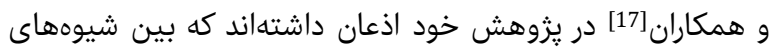

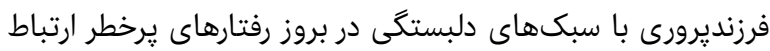

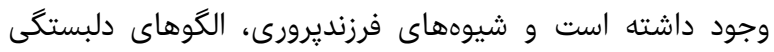

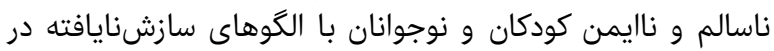

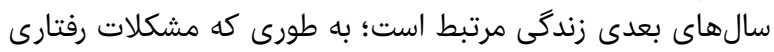

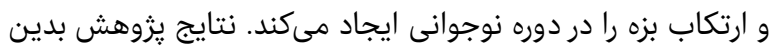

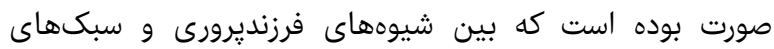

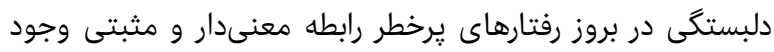

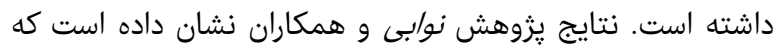

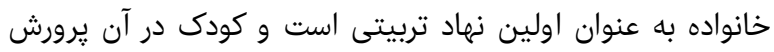

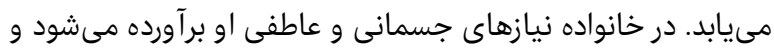

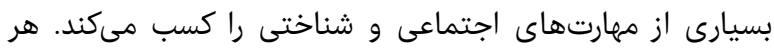

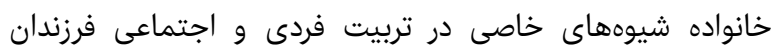

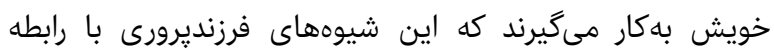

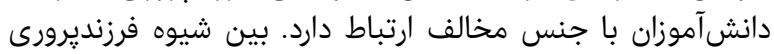

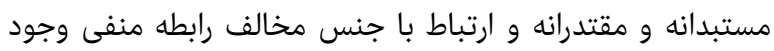

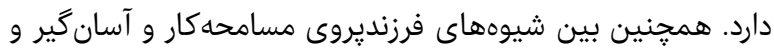

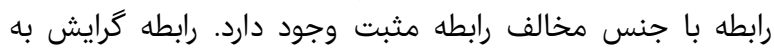

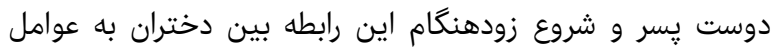

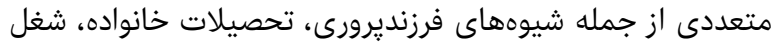

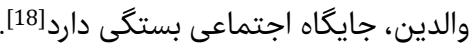

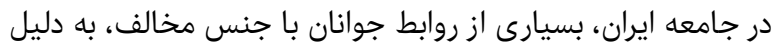

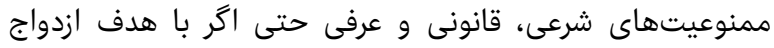

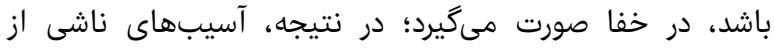

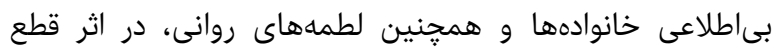

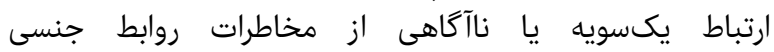

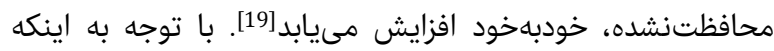

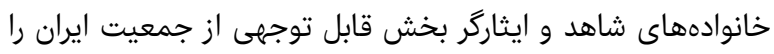

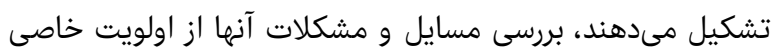

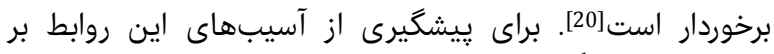

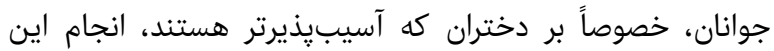

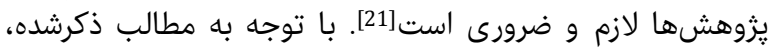




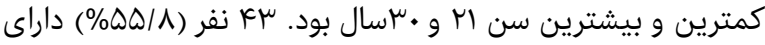

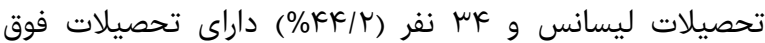

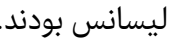

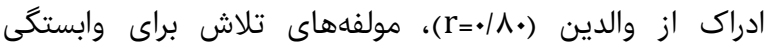
(r=./AV)

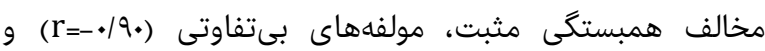

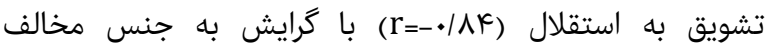

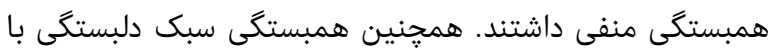

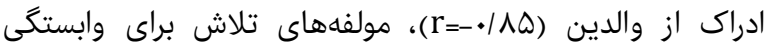
بr=-•/9T)

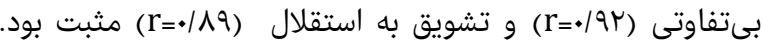

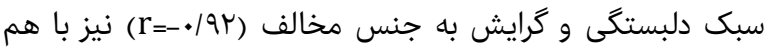

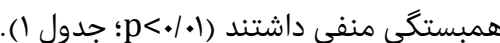

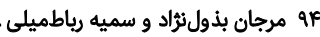

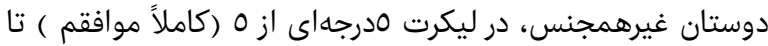

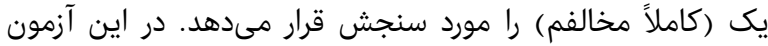

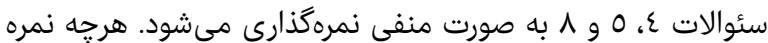

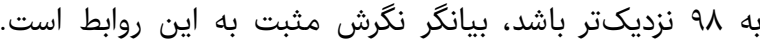

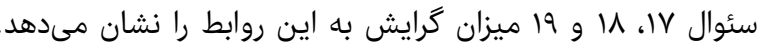

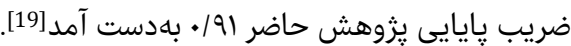

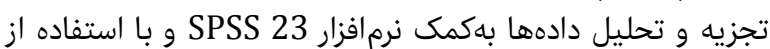

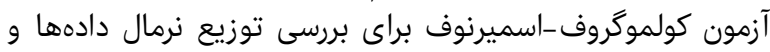

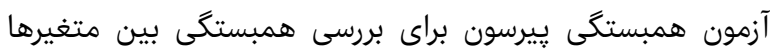

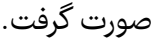

يافتهها

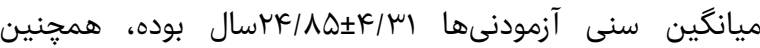

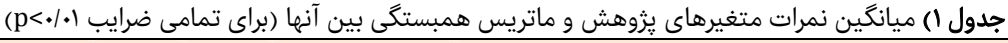

\begin{tabular}{|c|c|c|c|c|c|c|c|}
\hline 7 & 0 & $\varepsilon$ & $\mu$ & $r$ & 1 & نمرات & متغيرها \\
\hline & & & & & 1 & $V / r q \pm \psi / \varepsilon r$ & 1- بى \\
\hline & & & & 1 & . / & 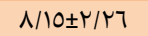 & r- تشويق به استقلال \\
\hline & & & 1 &.$- / 17$ &.- .190 & $\left.V / \Lambda \mu_{ \pm} \Psi /\right\urcorner \Lambda$ & "ـ - تلاش براى وابستخى \\
\hline & & 1 & $\cdot / 1 \Lambda$ &.- .190 &.$- / 1 \mathrm{~A}$ & $1 N / 9 r \pm \Psi / 7 V$ & ع - رابطه عاطفى \\
\hline & 1 &.$- / 9 \pi$ & $-.99 r$ & .119 & . $/ 9 r$ & $\varepsilon \cdot / \varepsilon 0 \pm 0 / \mu \varepsilon$ & 0- سبك دلبستگىى \\
\hline 1 &.$- \cdot 110$ & .119 & .110 &.$- / 199$ &.$- / \mathrm{VT}$ & $\varepsilon r / r \cdot \pm 1 / \Lambda \Lambda$ & 7ـ ادراك از والدين \\
\hline .11. &.$- / 94$ & .191 & ./AV & $-\cdot / \Lambda \varepsilon$ & -.19 & $V r / \Lambda \cdot \pm 9 / \Lambda \Lambda$ & V-كرايش به جنس مخالف \\
\hline
\end{tabular}

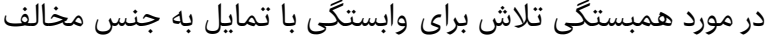

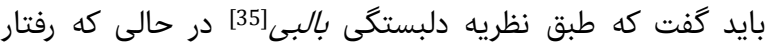

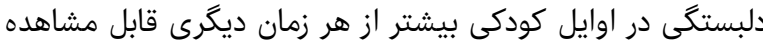

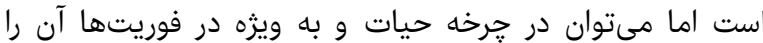

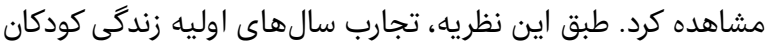

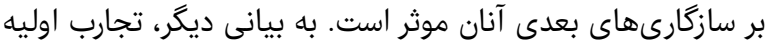

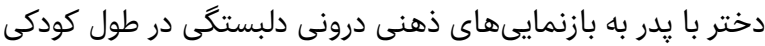

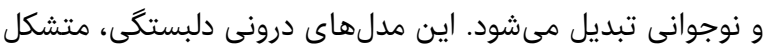

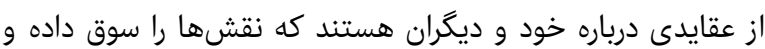

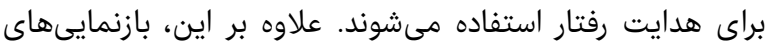

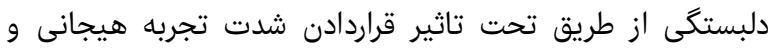

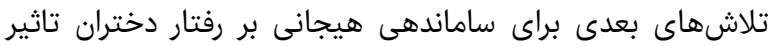

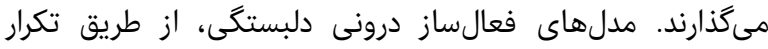

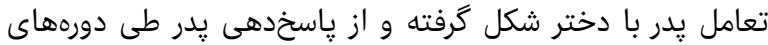

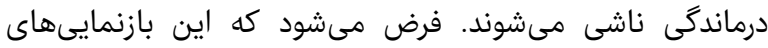

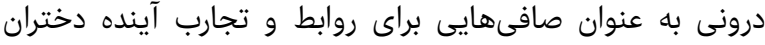

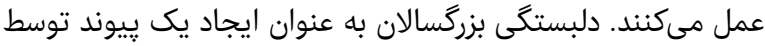

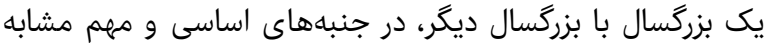

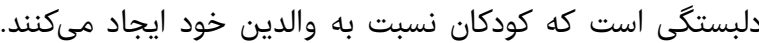

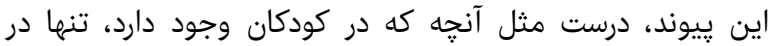

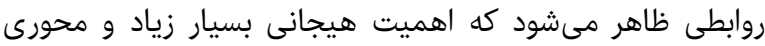

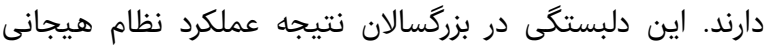

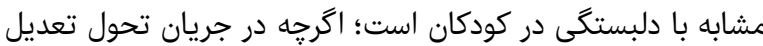

شده است[36].

در تبيين همبستگى بين تشويق به استقلال و تمايل به ارتباط با

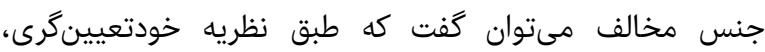

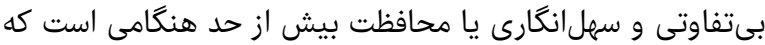

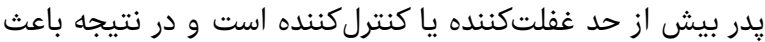

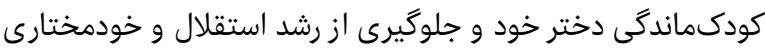

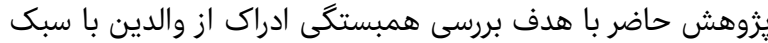

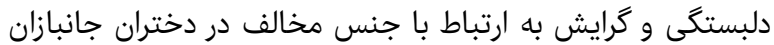

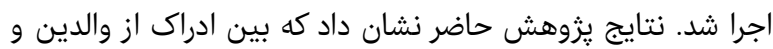

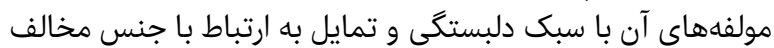

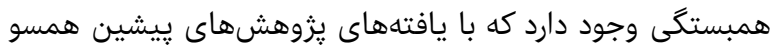

بود [9-18, 29-32.

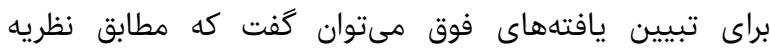

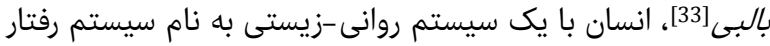

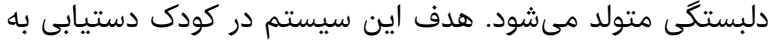

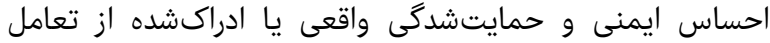

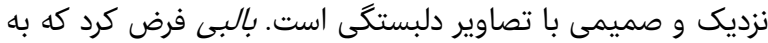

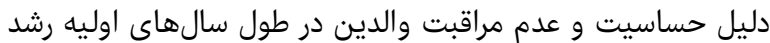

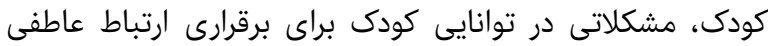

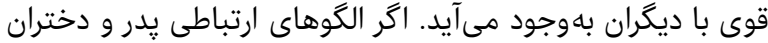

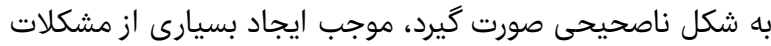

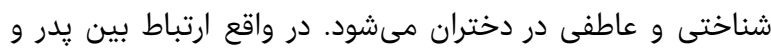

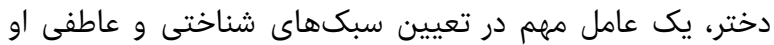

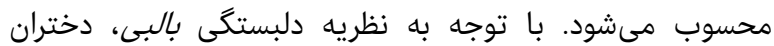

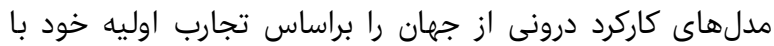

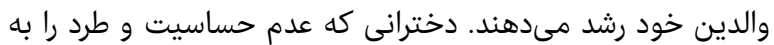

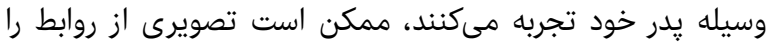

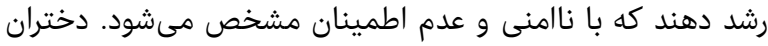

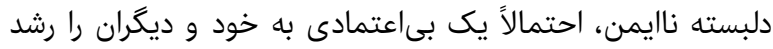

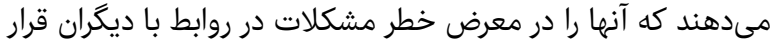

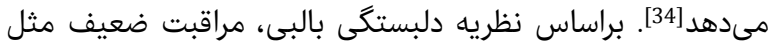

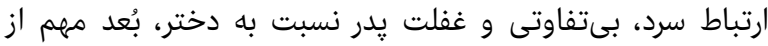

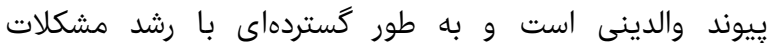
روانشناختى مرتبط هستند. 


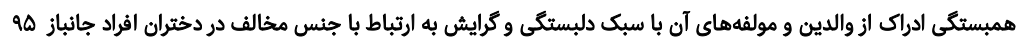

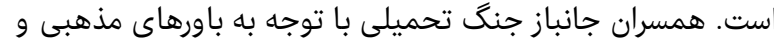

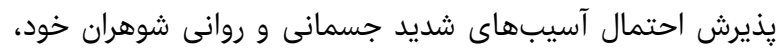

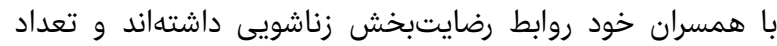

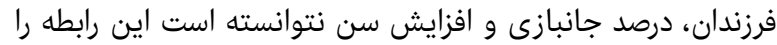
خدشهدار سازد.

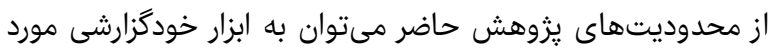

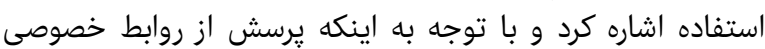

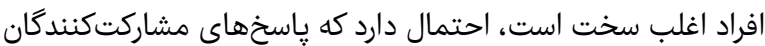

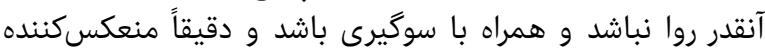

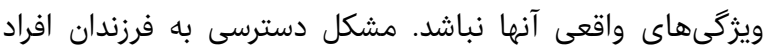

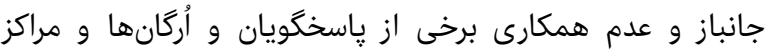

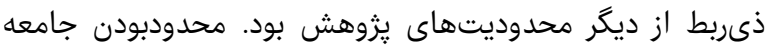

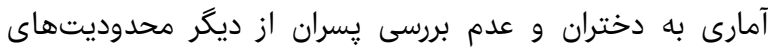

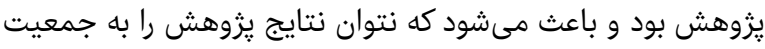

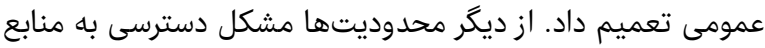

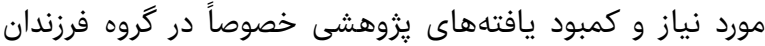

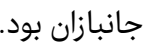
با توجه به تجربه جمعآورى دادهها و تحليل نتايج، پنانجه اين

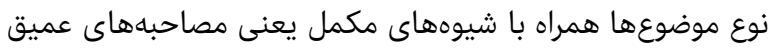

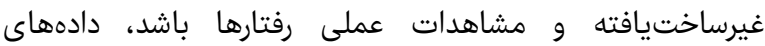

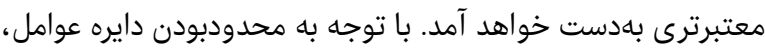

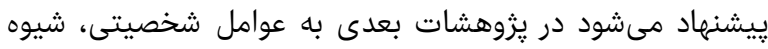

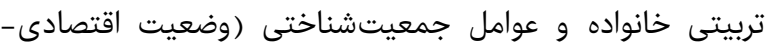

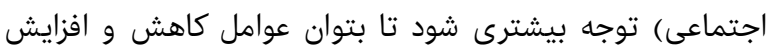

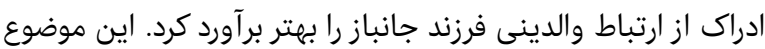

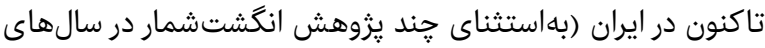

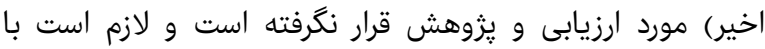

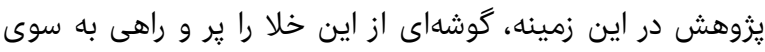

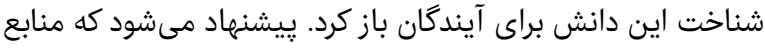

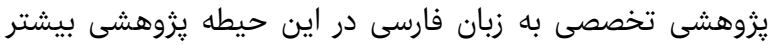

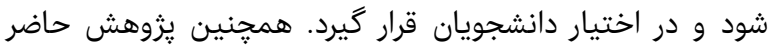

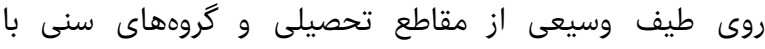

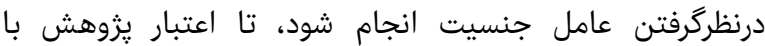
اطمينان بيشترى برآورد شود.

\section{نتيجه}

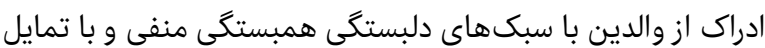

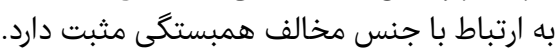

تشكر و قدردانى: از كليه فرزندان جانبازان و تمامى افرادى كه در

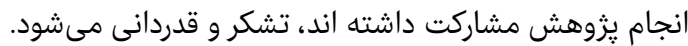

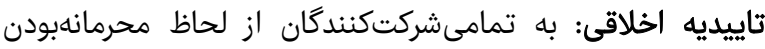

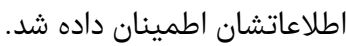

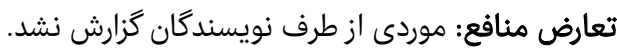

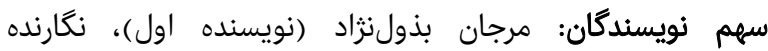

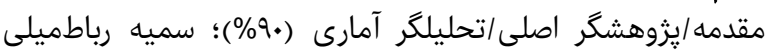

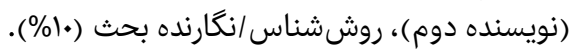

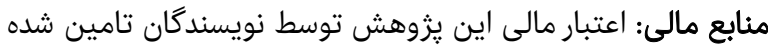
است.

منابع

1- Ahangarcani M, ZadehMohammadi A, Heidari M, Talebi M. The relationshib between marital values and
در او مىشود[37]. در اين نوع نكرشها، يدر تلاش بيشترى براى

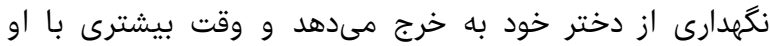

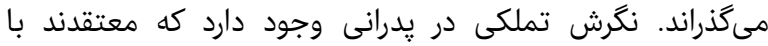

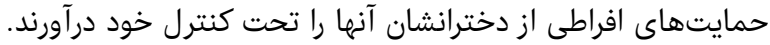

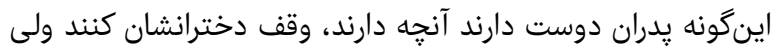

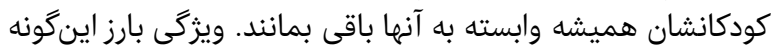

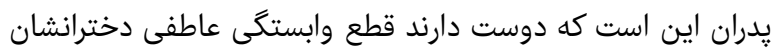

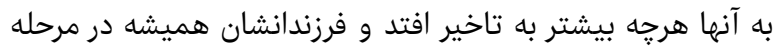
طفوليت باقى بمانند.

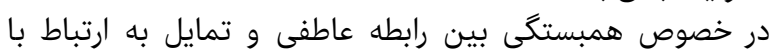

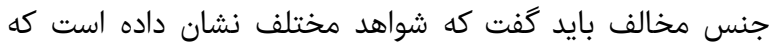

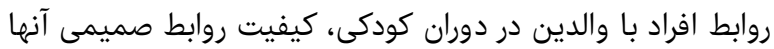

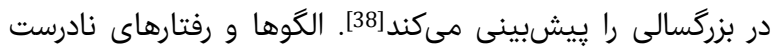

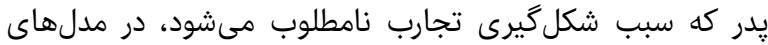

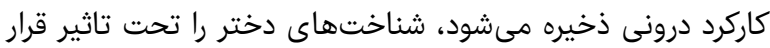

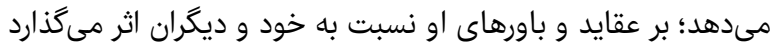

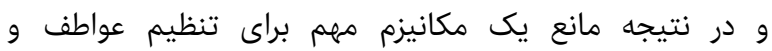

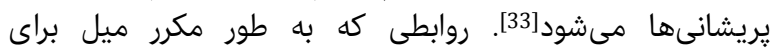

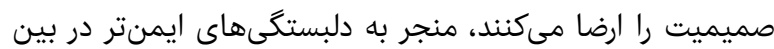

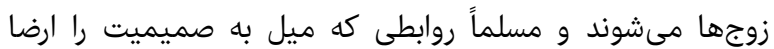

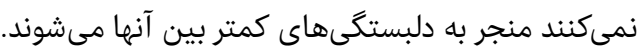

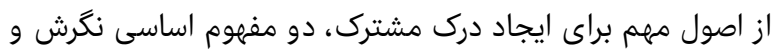

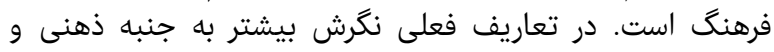

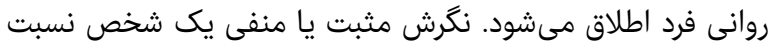

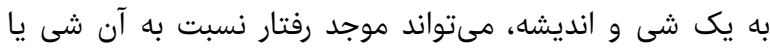

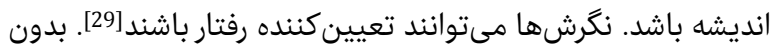

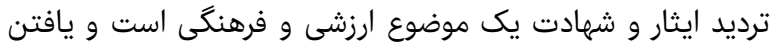

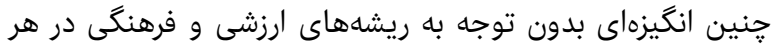

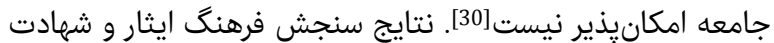

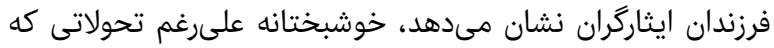

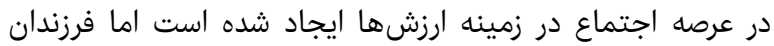

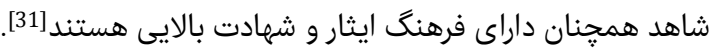

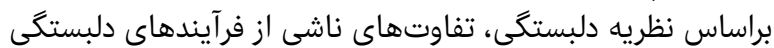

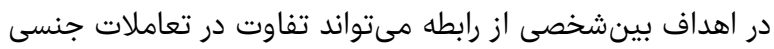

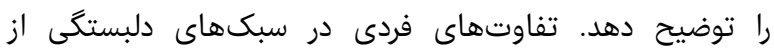

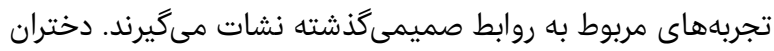

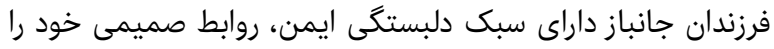

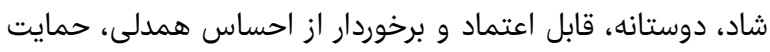

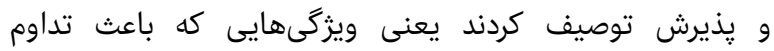

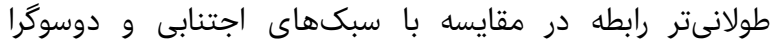

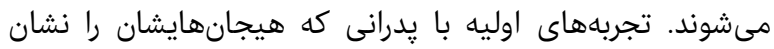

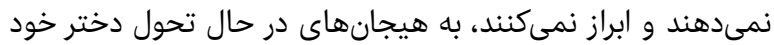

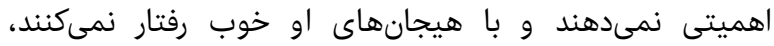

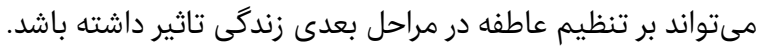

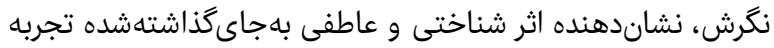

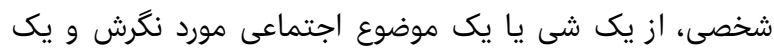

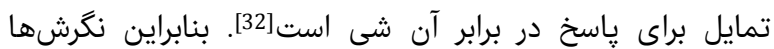

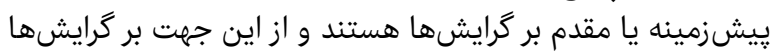

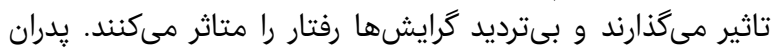

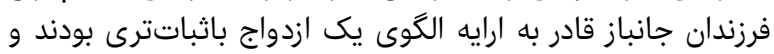

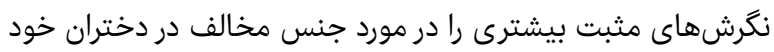

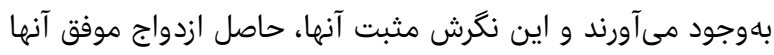


adolescents [Dissertation]. Tabriz: Tabriz University of Medical Sciences and Health Services; 2014. [Persian]

18- Navabi F. Rooting of prevalence of early relationship between girls and the opposite sex according to the parenting method [Dissertation] Tabriz: Tabriz University of Medical Sciences and Health Services; 2011. [Persian]

19- Pahlavan Zadeh F, Refahi J. The effectiveness of conflict resolution skills in tends to be the opposite sex in single girls. J Educ Couns Psychother. 2016;5(17):47-58. [Persian]

20- Moradi A, Abedi A, Rabbani R, Gholami Renani F. Meta-analysis results of research on the mental health level of sacrificer and martyr families. J Psychol. 2010;14(1):18-38. [Persian]

21- Khalaj Abadi Farahani F, Mehryar AM. Studying the role of family in communication with the opposite sex before marriage in female students in Tehran. J Fam Res. 2013;6(24):449-68. [Persian]

22- Khalatbari J. Statistics and research methodology. Tehran: Pardazesh; 2010. [Persian]

23- Mirza Nasrollah Shirazi F. Comparison of parenting pattern, tolerance of ambiguity and selective attention in patients with obsessive-compulsive disorder with nonaffected individuals [Dissertation]. Tehran: Islamic Azad University; 2010. [Persian]

24- Liss M, Timmel L, Baxley K, Killingsworth P. Sensory processing sensitivity and its relation to parental bonding, anxiety, and depression. J Personal Individ Differ. 2005;39(8):1429-39.

25- Qanadei F, Abdollahi MH. The relationship between perceptions of parental behavior and early maladaptive schemas. Clin Psychol Stud. 2014;4(16):129-51. [Persian]

26- Besharat M, Bazazian S. Attachment styles, illness perception and quality of life in patients with type I diabetes. Contemp Psycol. 2010;5(1):3-11. [Persian] 27- Rezapour Mirsaleh Y. The relationship between attachment styles and father-child conflict in warfare victims' children: Investigate the role of secondary trauma as a mediator. J Counsel Res. 2016;15(57):81100. [Persian]

28- Zareshahabadi A, solimani Z. Factors related to student's tendency towards premarital relations in Yazd University. Soc Welf. 2012;12(45):391-421. [Persian]

29- Rafi Pour F. Change of values in cinema and newspapers. Cult Res Lett. 1999;14 \& 15:5-30. [Persian] 30- Zokaei MS. Wanting for others and sociology: Reviewing theories. Culture. 1997;22 \& 23:275-96. [Persian]

31- Zahiri $\mathrm{H}$. The attitudes children of sacrificer and martyr to the culture of martyrdom. Soc Sci Bull. 2008;2(1):154-77. [Persian]

32- Salimi J, Karimi M. Survey the effect of Lazarus's theory's instruction on the attitude change toward Choice of spouse among female students. Fam Counsel Psychother. 2012;2(1):512-33. [Persian]

33- Roelofs J, Onckels L, Muris P. Attachment quality and psychopathological symptoms in clinically referred adolescents: The mediating role of early maladaptive schema. J Child Fam Stud. 2013;22(3):377-85.

34- Kawabata Y, Alink LRA, Tseng WL, van IJzendoorn MH, Crick NR. Maternal and paternal parenting styles associated with relational aggression in children and adolescents: A conceptual analysis and meta-analytic review. Dev Rev. 2011;31(4):240-78. family structure in male and female married individuals. Behav Sci. 2014;12(1): 4457. [Persian]

2- Sternberg SJ, Davila J, Finchman F. Adolescent marital expectations and romantic experlences: Association with perception about parental conflict and attachment security. J youth Adolesc. 2006;35(3):333-48.

3- Khodayari Fard M. Comparison of relationship between religious attitude and paternal relations with social adjustment in veterans and normal children in Tehran. J Psychol. 2004;8(4):372-88. [Persian]

4- Fakhri Z, Danesh E, Shahidi SH, Saliminia A. Quality of value systems and self-efficacy beliefs in offspring's of veterans and non-veterans fathers. J Appl Psychol. 2013;6(4):25-42. [Persian]

5- Ramerkha S, Bell ML, Paul C, Dickson N, Moffitti, TE, Caspi A. Childhood behavior problems linked to sexual risk taking in young adulthood: A birth cohort study. J Am Acad Child Adolesc Psychiatry. 2007;46(10):1272-9. 6- Alexander M, Garda L, Kanade S, Jejeebhoy S, Ganatra B. Correlates of premarital relationships among unmarried youth in Pune district, Maharashta, India. Int Fam Plan Perspect. 2007;33(4):150-9.

7- Khalej Abadi Farahani F, Mehriar AH. Investigating the role of family in communication with the opposite sex before marriage in female students in Tehran. J Fam Res. 2010;6(4):449-68. [Persian]

8- Azimi Hashemi M, AzamKari F, Bigonah M, Rezamanesh F. Values, Attitudes and Models of the Youth Actions in Choosing Spouses and Premarital Relations; Case of Study: Students of universities of Khorasan Razavi Province. 2015;8(29):179-212. [Persian]

9- Potard C, Courtois R, Réveillère Ch, Courtois A. The relationship between parental attachment and sexuality in early adolescence. Int J Adolesc Youth. 2017;22(1):4756.

10- Maximo SI, Carranza JS. Parental attachment and love language as determinants of resilience among graduating university students, SAGE Open. 2016;6(1):1-11.

11- Einav M. Perceptions about parents' relationship and parenting quality, attachment styles, and young adults' intimate expectations: A cluster analytic approach. J Psychol. 2014;148(4):413-34.

12- Millings A, Walsh J, Hepper E, O’Brien M. Good Partner, good Parent responsiveness mediates the link between romantic attachment and parenting style. Pers Soc Psychol Bull. 2013;39(2):170-80.

13- Seiffge-Krenke I, Overbeek G, Vermulst A. Parentchild relationship trajectories during adolescence: Longitudinal associations with romantic outcomes in emerging adulthood. J Adolescence. 2010;33(1):159-71. 14- Hall SS. Paternal influences on daughters' heterosexual relationship socialization: Attachment style and disposition toward marriage. Fam Sci Rev. 2009;14(2):1-17.

15- Kamel Abbasi AR, Tabatabaei SM, Aghamohammadiyan Sharbaf H, Karshki H. Relationship of attachment styles and emotional intelligence with marital satisfaction. Iran J Psychiatry Behav Sci. 2016;10(3):2-10.

16- Mahmoodi N, Naziri Gh, Zarenezhad M. Attachment Styles in Sufferers of Gender Identity Disorder in Fars Province in 2014. Jentashapir J Health Res. 2016;7(1):17.

17- Khosravi M. Relationship between parenting styles and attachment styles in high risk behaviors in 
qv همبستى ادراك از والدين و مولفههاى آن با سبك دلبستگى و كرايش به ارتباط با جنس مخالف در دختران افراد جانباز

inadequate parental bonding and peer victimization for adolescent mental health. J Adolesc. 2007;30(5):801-12.

35- Bowlby J. Attachment and loss: Separation: Anxiety 38- Darling N, Cohan C, Burns A, Thompson L. Withinfamily conflict behaviors as predictors of conflict in adolescent romantic relations. J Adolesc. 2007;31(6):67190. and anger. New York: Basic Books; 1973.

36- Byng-Hall J. Rewriting family scripts: Improvisation and systems change. New York: Guilford Press; 1995.

37- Rigby K, Slee PT, Martin G. Implications of 